\title{
The Effect of Heparinoid as Systemic Prophylactic Anticoagulants on COVID-19 Patient Mortality and Its Safety Profiles: A Systematic Review and Meta-Analysis
}

\author{
Ariska Deffy Anggarany ${ }^{1}$, Rani Sauriasari ${ }^{1 *}$, Muhammad Alkaff ${ }^{2}$, Famila Takhwifa ${ }^{1}$, \\ Hayatun Nufus ${ }^{2}$, Diana Paramita ${ }^{2}$ \\ ${ }^{1}$ Faculty of Pharmacy, Universitas Indonesia, Depok, Indonesia \\ ${ }^{2}$ Department of Internal Medicine, Persahabatan Central General Hospital, Jakarta, Indonesia
}

\begin{abstract}
Coagulopathy is one of the complications of COVID-19 and is associated with a higher risk of mortality. However, evidence regarding the effectiveness and safety of anticoagulant therapy in various doses among COVID-19 patients is limited. This systematic review and meta-analysis aims to review and explore the effect of using heparinoids as a systemic anticoagulant at prophylactic doses on mortality in COVID-19 patients. Systematic searches were conducted of various databases (Pubmed, ScienceDirect, SpringerLink, Scopus, and ProQuest) covering the period 2019-2021. We assessed the quality of the articles using the STROBE checklist. Studies with a high risk of bias were excluded before pooled effect size was synthesized with $95 \%$ confidence intervals $(\mathrm{CI})$ using random-effects models. From the 12 identified studies $(\mathrm{N}=8,968)$, six observational studies $(\mathrm{N}=7,176)$ were involved in the meta-analysis. The studies reviewed in the paper used a retrospective cohort design in various settings. The pooled effect size of mortality comparing prophylactic anticoagulant and no anticoagulant in three studies showed that there was an association between using prophylactic anticoagulant and a lower risk of in-hospital mortality (pooled $\mathrm{OR}=0.47 ; 95 \%$ CI $0.19-0.76$ ). A prophylactic dose of heparinoid anticoagulant was also associated with lower mortality (pooled $\mathrm{OR}=0.51 ; 95 \% \mathrm{CI} 0.21-0.82$ ) and with lower bleeding events compared to intermediate-to-therapeutic dose anticoagulants. Administration of heparinoid anticoagulants at prophylactic doses was associated with reduced mortality risk in hospitalized COVID-19 patients. Due to the increased risk of bleeding with therapeutic doses, the use of prophylaxis anticoagulant is suggested in COVID-19 patients who are not critically ill.
\end{abstract}

Keywords: anticoagulants; prophylaxis; covid-19; mortality; therapeutic safety

*corresponding author

Email:rani@farmasi.ui.ac.id

\section{INTRODUCTION}

In late December 2019, the highly infectious coronavirus rapidly spread worldwide and caused coronavirus disease 2019 (COVID-19). Severe respiratory illness can be induced by the severe acute respiratory syndrome coronavirus-2 (SARS-CoV-2) (Zhu et al., 2020). Several studies have shown that SARS-CoV-2 virus infection is linked to substantial coagulation system activity, leading to prothrombotic conditions (AbouIsmail et al. 2020; Bikdeli et al., 2020). Coagulopathy is one of the COVID-19 complications that can cause mortality (chiefly in patients with severe COVID-19) (Zhou et al., 2020). It can lead to vascular complications, especially venous thromboembolism (VTE), also known as Deep Vein Thromboembolism (DVT) or Pulmonary Embolism (PE), which have been widely reported to have significant implications for the clinical outcome of COVID-19 patients (Klok et al., 2020; Langer et al., 2020). A cohort study of 191 patients with COVID-19 reported that $50 \%$ of in-hospital deaths were in individuals who had coagulopathy (Zhou et al., 2020). The occurrence of coagulopathy as part of the systemic inflammatory response syndrome is a frequent feature of severe COVID-19. Almost 20\% - 50\% of COVID-19 hospitalized patients had hematologic abnormalities in coagulation testing, and up to $71.4 \%$ of COVID-19 deaths are caused by Disseminated Intravascular Coagulopathy (DIC) (Gómez-Mesa et al, 2021). Prophylactic anticoagulant has been linked to a reduction in the incidence of thrombosis and death in patients; as a result, it can help prevent and decrease COVID-19 mortality (Langer et al., 2020). The use of Low Molecular Weight Heparin (LMWH) as an initial anticoagulant in COVID-19 patients has been shown to lower mortality by $48 \%$ after 7 days, and $37 \%$ after 28 days (Gómez-Mesa et al., 2021). Therefore, managing coagulopathy, including thromboembolic prophylaxis and anticoagulant treatment, is essential for optimizing specific therapy and reducing mortality. Guidelines from the International Society of Thrombosis Haemostasis (ISTH) recommend prophylactic systemic anticoagulant 
using LMWH among all hospitalized patients, regardless of their coagulopathy status (Thachil et al., 2020). Heparin is recommended because, apart from being an anticoagulant, it theoretically acts as an antiinflammatory and antiviral (Hippensteel et al., 2020). Systemic heparinoid anticoagulants, both Unfractionated Heparin (UFH) and LMWH, have been reported to be more beneficial than oral anticoagulants for use in COVID-19 hospitalized patients. There is a higher risk of interaction between antivirals or other drugs with oral anticoagulants, rather than with systemic anticoagulants (Weeks, Connors, \& Connell, 2021).

Several clinical studies regarding the assessment of the effect of prophylactic anticoagulants are ongoing. Tang et al. (2020) reported that prophylactic anticoagulants had a greater survival rate than no anticoagulant in a severely ill patient COVID-19, elevated D-dimer values, and coagulopathy (Tang et al., 2020). Dosage increases may be necessary because the incidence of thrombosis is still high, despite prophylactic anticoagulants (Patel et al., 2020). However, in-hospital mortality in prophylactic and therapeutic doses has been reported to be not significantly different, while therapeutic doses have increased bleeding complications more than prophylactic doses (Klok et al., 2020; Nadkarni et al., 2020). Therefore, it can be seen that the role of anticoagulants at higher doses remains controversial today.

For this reason, we accomplish a systematic review of several previous studies with more recent time on the effect of the prophylactic use of systemic anticoagulants on all-cause mortality in hospitals. We assessed the safety of therapy related to bleeding to provide clinical insight and consider using systemic anticoagulants in the treatment of COVID-19 patients in the hospital. A previous meta-analysis has asessed the association of administration of anticoagulants in various types (heparinoids, vitamin $\mathrm{K}$ antagonists, direct anticoagulants, etc.) on all-cause mortality in hospitalized COVID-19 patients (Flumingnan et al., 2020). However, in our study, we focus on the systemic use of heparinoid anticoagulants, i.e. unfractionated heparin and low molecular weight heparin, and pentasaccharides (a synthetic and selective anticoagulant drug similar to low molecular weight heparin), as heparin is more recommended for inpatients due to safety reasons related to drug interactions (heparin does not interact with other drugs administered to hospitalized COVID-19 patients). We hope this systematic review and meta-analysis will help clinicians when considering the use of heparinoid as a systemic prophylactic anticoagulant for COVID-19 patients.

\section{METHODS}

The systematic review and meta-analysis were performed according to the Preferred Reporting Items for Systematic Reviews and Meta-Analysis (PRISMA) Guideline (Figure 1) (Page et al., 2021).

\section{Search Procedure}

We organized a systematic review to analyze and synthesize some of the latest studies regarding assessment of the effect of prophylactic anticoagulants. A systematic search was performed of the online database of Pubmed, SpringerLink, ScienceDirect, Scopus, and ProQuest to discover compatible articles. The primary search was restricted to articles published during the COVID-19 pandemic from 2019 to 2021. The language was limited to English, and we used a specific key search term ("Anticoagulant" OR "LMWH" OR "Heparin") AND ("Prophylaxis" OR "Prophylactic" OR "Subtherapy") AND "COVID" AND "Mortality." Employing these data, a systematic review was conducted on the effect of heparinoid anticoagulant at prophylactic doses on mortality amongst COVID-19 patients. Duplicate studies were excluded from this meta-analysis. We used EndNote tools to help that process. Two investigators independently explored the literature, screened titles and abstracts, and carefully evaluated full text to analyze potentially eligible studies. In case of disagreement, it was consulted and solved by the consensus of all authors.

\section{Criteria for Inclusion and Exclusion}

The patients' inclusion and exclusion criteria were established by the Population, Intervention, Comparison, Outcomes (PICO) formula. The population comprised patients with confirmed COVID-19 with RT-qPCR results and those undergoing hospitalization (any patients COVID-19 as long they were hospitalized). Intervention referred to heparinoid anticoagulants at prophylactic doses (both LMWH and UFH), while the comparison was based on patients not being given systemic prophylactic anticoagulants or heparionoid anticoagulants at intermediate-to-therapeutic doses. The outcomes related to establishing the clinical outcome of using systemic anticoagulants in the prophylactic dose and their effect on patient mortality. On the other hand, the exclusion criteria were: (1) non-original articles (review articles); (2) non-clinical studies; (3) non-COVID-19 patients; (4) patients not hospitalized; and (5) no comparison made between heparinoid anticoagulants at prophylactic and therapeutic doses or between prophylactic heparinoid anticoagulant and without anticoagulant treatment.

\section{Schemes and Search Results}

5,186 articles were discovered based on an online database search using specified keywords, including 
259 from Pubmed, 708 from SpringerLink, 1,058 from ScienceDirect, 1,248 from Scopus, and 1,903 from ProQuest. After screening the title, abstract and full-text articles, 12 articles were found to match the stipulated inclusion criteria, and the review process was resumed (included in qualitative synthesis). The search results schemes are summarized in Figure 1.

\section{Data Extraction and Quality Assessment}

The extracted data for characteristics comprised the author's name and year, inclusion/exclusion criteria, population characteristics (age, sex), setting, followup period, and the dosage of anticoagulant treatment. All-cause mortality was documented for the primary outcomes, and for the treatment, safety assessment was bleeding. We collected and provided results in the form of narration and a table.
The authors used the STROBE checklist to assess the quality and authenticity of the observational researches (von Elm et al., 2014). This checklist STROBE covers 22 quality assessment domains. Items with fully reported detailed data received a score of 1 , items with partially reported detailed data received a score of 0.5 , and items with not reported detailed data received a score of 0 . As a result, each study had a STROBE score rate ranging from 0 to 22 . Two authors reviewed each study independently and scored them according to the STROBE checklist. All of the disagreement was consulted and solved by the consensus of all authors.

\section{Data Synthesis}

We planned to initiate meta-analyses only where this was consequential, that is, if the treatments, subject, and the underlying clinical investigation were similar enough for

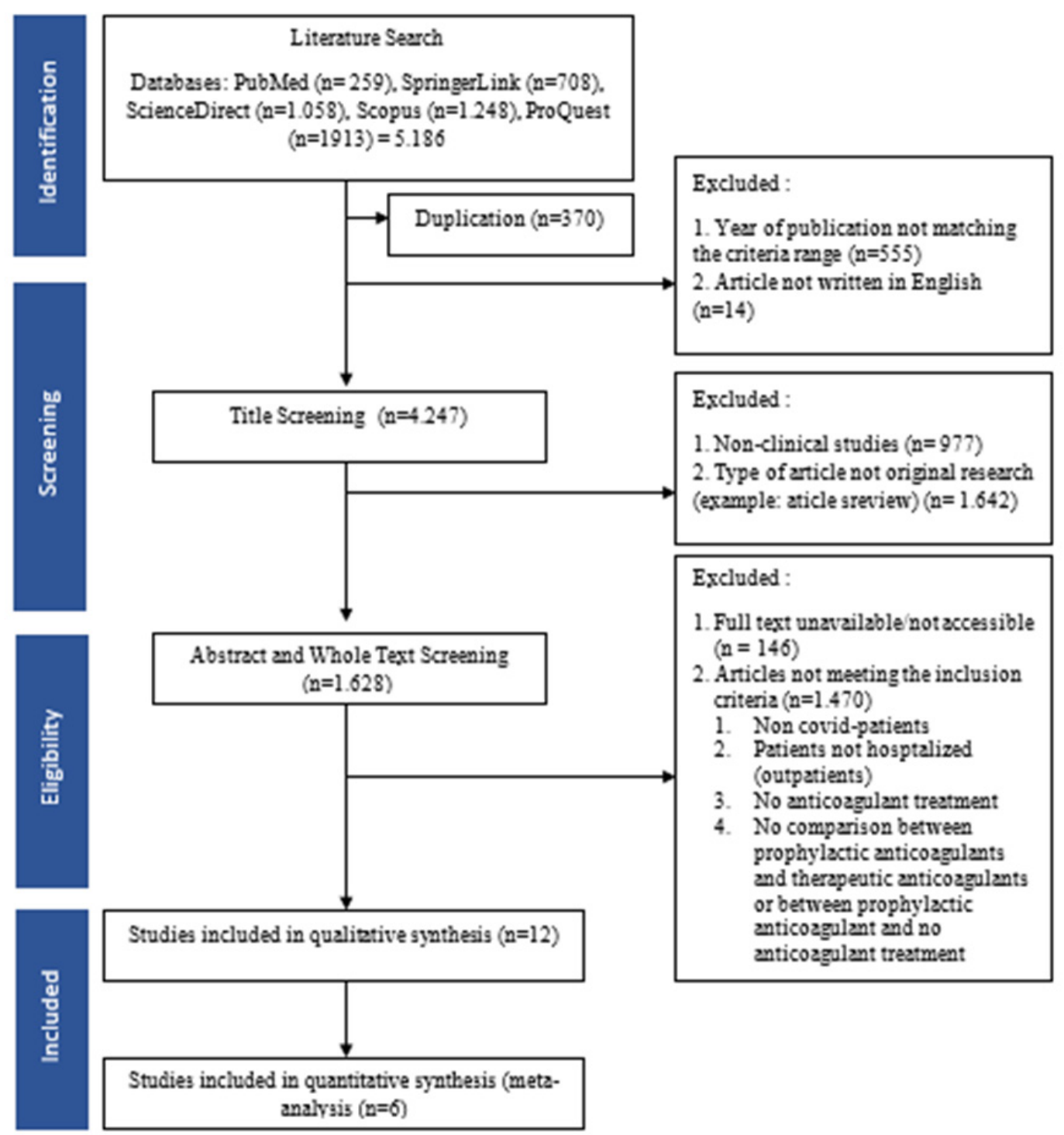

Figure 1. Literature Search Scheme with PRISMA flow 
pooling to succeed. The comparative meta-analysis did not include results from studies with no outcome event, studies with poor methodological quality, or studies with a high risk of bias. The main meta-analysis included all studies that described adjusted estimates of the effects of prophylaxis anticoagulant on in-hospital mortality compared to no anticoagulant use in COVID-19 patients who were hospitalized. We also added to the metaanalysis the effect estimate of the thromboprophylaxis dosage compared to different dose (intermediate-totherapeutic) anticoagulants.

When feasible, we conducted meta-analysis and forest plot using a Microsoft Excel Spreadsheet to calculate a summary effect size (Neyeloff, Fuchs, \& Moreira, 2012). By entering step-by-step formulas, statistics such as $\mathrm{Q}$ and $\mathrm{I}^{2}$ can be calculated using basic arithmetic operations. Heterogeneity was determined using the $\mathrm{I}^{2}$ value, and low heterogeneity is defined as $\mathrm{I}^{2}$ less than $50 \%$, moderate heterogeneity as $\mathrm{I}^{2}$ between $50 \%$ and $75 \%$, and high heterogeneity as $\mathrm{I}^{2}$ higher than $75 \%$. A random-effect model would be used to report the result of heterogeneous data. If meta-analysis is not possible, for example if there are substantial clinical, methodological, or statistical heterogeneity across studies prevented the pooling of data, the results will be synthesized narratively.

\section{RESULTS}

\section{Characteristics of the Included Studies}

The review was compiled systematically to examine the development of the latest studies (2019-2021) regarding the impact of prophylactic systemic anticoagulants on COVID-19 patients' mortality through clinical reviews. A systematic method was utilized to help readers easier to understand the content and to avoid bias. Figure 1 shows the study selection process. In the initial literature search using predetermined keywords, we identified a total of 5,186 articles. After screening of the the title, abstract and full text, 12 articles ( $\mathrm{N}=8,968$ patients) were selected, which were considered suitable because they met the inclusion criteria set. Furthermore, only 6 studies $(\mathrm{N}=7,176$ patients) continued for quantitative synthesis by meta-analysis. The other 6 studies had heterogeneous characteristics, different settings, and outcome units, so meta-analysis could not be carried out

All of the researches are retrospective observational studies, and most are from European countries. All the studies were based on a hospital setting, including ICU and medical wards. Three studies focused on ICU patients (Helms et al., 2021; Jonmarker et al., 2020; Zheng et al., 2021). The nine other studies were based on an all-hospitalized patient setting, apart from Pesavento et al. (2020), who only used a medical ward patient setting. In general, the study was conducted by collecting retrospective data on treatment, outcome, the severity of COVID-19 and comorbidities, obtained from patients' medical records, with hospital mortality defined as any death that occurred during hospitalization for whatever reason. In most of studies, the primary endpoint was “all-cause death" (Table 2).

In the 12 studies, the sample size ranged between 150 and 5000 patients; The smallest sample size was 152 patients in the study of Jonmarker et al. (2020) and the most sample used was 4,297 patients found in the study of Rentsch et al. (2021). All the studies involved adult men and women ( $\geq 18$ years), with the majority being male $(>55 \%)$, apart from the study of Shen et al. (2021), in which the percentage of men was lower $(49.3 \%)$ than that of women (Shen et al., 2021) The median age for the study was 63 , with the age range varying from 54 to 73.7 years (Table 1).

Table 1 displays the overall quality assessment score of the included studies using the STROBE guidelines (von Elm et al., 2014). The included studies' total scores varied from 17 to 21 , indicating that the studies' quality ranged from moderate to high. In general, the quality of the studies in this systematic review is suitable based on the scores obtained. A summary of the overall STROBE checklist assessment is available in the Supplementary file.

Qualitative Review: Association of Anticoagulant Treatment with mortality

\section{Anticoagulant prophylaxis versus non-use of anticoagulants}

In the 12 studies that were reviewed, four studies compared prophylactic anticoagulant versus no anticoagulant with their related with a decreased risk of in-hospital mortality patients with COVID-19 (Albani et al., 2020; Rentsch et al., 2021; Shen et al., 2021; Zheng et al., 2021). Of these four studies, all used an LMWH systemic anticoagulant (enoxaparin) at a prophylactic dose of $40 \mathrm{mg}$ daily (Albani et al., 2020; Shen et al., 2021) or $100 \mathrm{IU}$ Axa/kg once per day (Zheng et al., 2021). In contrast, Rentsch et al. (2021) did not list the prophylactic dose of enoxaparin used (Albani et al., 2020) (Table 2). Overall, the four studies $(\mathrm{N}=6,375$ patients) showed that prophylactic heparinoid anticoagulant (LMWH/enoxaparin) was related with decreased odds of mortality in patients with COVID-19: adjusted OR $0.53,95 \%$ CI $0.40-0.70 ; 1,376$ participants (Albani et al., 2020); adjusted HR 0.69, 95\% CI 0.610.77; 4,297 participants (Rentsch et al., 2021); adjusted OR 0.20 , 95\% CI 0.09-0.46; 525 participants (Shen et al., 2021); unadjusted OR 0.60; 95\% CI 0.32-1.01; 180 participants (Zheng et al., 2021). 
Table 1. Characteristics of the included studies

\begin{tabular}{|c|c|c|c|c|c|c|c|c|}
\hline Author & Year & Study Design & Country & COVID-19 Patient Type & $\mathbf{N}$ & Age & Male sex (\%) & $\begin{array}{l}\text { Quality } \\
\text { Analysis } \\
\text { Score }\end{array}$ \\
\hline Jonmarker et al. & 2020 & Retrospective cohort study & Sweden & ICU Patients & 152 & Median (IQR): 61 (52-69) & $125(82.2)$ & 19 \\
\hline Canola \& Saylan & 2020 & Retrospective study & Turkey & All COVID-19 inpatients & 154 & Median (IQR): 55 (48-66) & $96(62.34)$ & 18 \\
\hline Pesavento et al. & 2020 & Retrospective cohort study & Italy & Medical Wards Patients & 324 & $\begin{array}{l}\text { Median (IQR): } 71 \\
(59-82)\end{array}$ & $181(55.9)$ & 17 \\
\hline Albani et al. & 2020 & Retrospective cohort study & Italy & All COVID-19 inpatients & 1376 & Median (IQR) : 68 (58-76) & $379(67.15)$ & 20 \\
\hline Grandone et al. & 2021 & Retrospective cohort study & Italy & All COVID-19 inpatients & 264 & Median (IQR): $72(58-80)$ & $149(56.4)$ & 19 \\
\hline Mennuni et al. & 2021 & Retrospective cohort study & Italy & All COVID-19 inpatients & 436 & $\begin{array}{l}\text { Mean (SD): } \\
71.2(15.6)\end{array}$ & $159(55.4)$ & 21 \\
\hline Shen et al. & 2021 & Retrospective cohort study & China & All COVID-19 inpatients & 525 & Median (IQR): 64 (52-72) & $259(49.3)$ & 20 \\
\hline Martinelli et al. & 2021 & Retrospective cohort study & Italy & All COVID-19 inpatients & 278 & $\begin{array}{l}\text { Median (IQR): } \\
59(49-67)\end{array}$ & $181(65.1)$ & 20 \\
\hline Zheng et al. & 2021 & Retrospective study & China & ICU Patients & 180 & Median (IQR): 64 (51-72) & $113(62.7)$ & 19 \\
\hline Farrar et al. & 2021 & Retrospective cohort study & $\begin{array}{l}\text { United } \\
\text { State }\end{array}$ & All COVID-19 inpatients & 803 & $\begin{array}{l}\text { Median (IQR): } \\
54(42-65)\end{array}$ & $446(55.5)$ & 19 \\
\hline Rentsch et al. & 2021 & Retrospective cohort study & $\begin{array}{l}\text { United } \\
\text { States }\end{array}$ & All COVID-19 inpatients & 4297 & Median (IQR): 68 (58-75) & 4015 (93.4) & 21 \\
\hline Helms et al. & 2021 & Bicenter cohort & France & ICU Patients & 179 & Median (IQR): 62 (51-70) & $130(72.6)$ & 18 \\
\hline
\end{tabular}

Abbreviations: ICU, Intensive Care Unit; IQR, Interquartile; SD, Standard Deviation; N, Number 
Table 2. Anticoagulant prophylaxis in COVID-19 mortality

\begin{tabular}{|c|c|c|c|c|c|c|}
\hline Author/Study & Intervention & Comparison & $\begin{array}{l}\text { Follow up } \\
\text { treatment follow } \\
\text { up }\end{array}$ & $\begin{array}{l}\text { Outcome } \\
\text { assessment }\end{array}$ & $\begin{array}{l}\text { Mortality } \\
\text { A vs. B (\%) }\end{array}$ & OR/HR \\
\hline $\begin{array}{l}\text { (Jonmarker et } \\
\text { al., 2020) }\end{array}$ & $\begin{array}{l}\text { Standard dose } \\
\text { thromboprophylaxis } \\
\text { (for ICU patients): } \\
2500- \\
5000 \text { IU dalteparin, } \\
\text { or 2500-4500 IU } \\
\text { tinzaparin. }\end{array}$ & 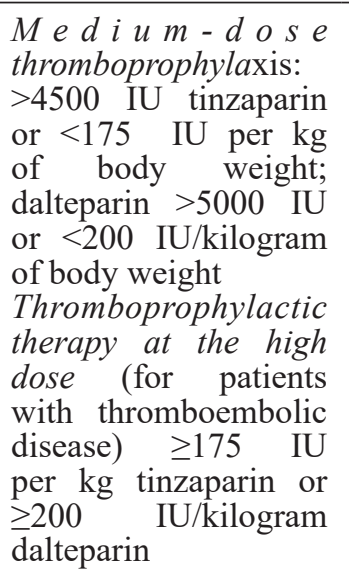 & $\begin{array}{l}\text { Patients were } \\
\text { monitored } \\
\text { until they died } \\
\text { or until the 28th } \\
\text { day after being } \\
\text { admitted to the } \\
\text { ICU }\end{array}$ & $\begin{array}{l}\text { The primary } \\
\text { outcome was } \\
\text { mortality at } \\
\text { 28-day }\end{array}$ & $\begin{array}{l}\text { Patients who received } \\
\text { high: lower : medium-dose } \\
\text { prophylactic }=13.5 \%: 25.0 \% \\
: 38.8 \%\end{array}$ & $\begin{array}{l}\text { In comparison to low-dose } \\
\text { thrombophylaxis, the HR of death for } \\
\text { high dose was } 0.33 \text { ( } 95 \% \text { CI } 0.13- \\
0.87 \text { ) and } 0.88(95 \% \text {.CI } 0.43-1.83) \text { for } \\
\text { medium dose. }\end{array}$ \\
\hline $\begin{array}{l}\text { (Canoglu } \& \\
\text { Saylan, 2020) }\end{array}$ & $\begin{array}{l}\text { prophylactic LMWH : } \\
0.5 \mathrm{mg} / \mathrm{kg} \text { twice daily }\end{array}$ & $\begin{array}{l}\text { therapeutic } \\
\text { Dose: } 1 \mathrm{mg} / \mathrm{kg} \text { twice } \\
\text { daily }\end{array}$ & $\begin{array}{l}\text { Patients were } \\
\text { tracked from the } \\
\text { time they were } \\
\text { admitted to the } \\
\text { hospital until the } \\
\text { earliest data on } \\
\text { outcomes were } \\
\text { available. }\end{array}$ & $\begin{array}{l}\text { In-hospital } \\
\text { mortality }\end{array}$ & $\begin{array}{l}44.9 \% \text { ( } 98 \text { patients }) \text { with } \\
\text { prophylactic } \\
\text { dose of LMWH died } \\
17.9 \% \text { ( } 10 \text { patients }) \text { of } 56 \\
\text { patients with therapeutic } \\
\text { Dose of LMWH died }\end{array}$ & $\begin{array}{l}\text { Prophylactic dose LMWH had a higher } \\
\text { risk of mortality than the therapeutic } \\
\text { dose (OR } 6.495 ; 95 \% \text { CI } 2.393-17.627 \text { ) }\end{array}$ \\
\hline $\begin{array}{l}\text { (Pesavento et } \\
\text { al., 2020) }\end{array}$ & $\begin{array}{l}\text { Prophylactic doses: } \\
\text { UFH 5000 UI TID, } 40 \\
\text { mg OD of LMWH, } 2.5 \\
\text { mg daily Fondaparinux }\end{array}$ & $\begin{array}{l}\text { Subtherapeutic doses: } \\
1 \mathrm{mg} / \mathrm{kg} \mathrm{BID} \text { of or } \\
0.5 \mathrm{mg} / \mathrm{kg} \mathrm{BID} \mathrm{of} \\
\text { LMWH, } 7.5 \mathrm{mg} \text { of } \\
\text { Fondaparinux }\end{array}$ & $\begin{array}{l}\text { Patients were } \\
\text { tracked from the } \\
\text { time they were } \\
\text { admitted to the } \\
\text { hospital until the } \\
\text { earliest data on } \\
\text { outcomes were } \\
\text { available. }\end{array}$ & $\begin{array}{l}\mathrm{O} \vee \text { e } r \text { a } 11 \\
\text { mortality }\end{array}$ & $\begin{array}{l}\text { Overall mortality rates were } \\
12.2 \text { per } 100 \text { people per month } \\
\text { in the prophylactic group and } \\
20.1 \text { per } 100 \text { in the (sub) } \\
\text { therapeutic group. }\end{array}$ & $\begin{array}{l}\text { Overall mortality rates were } \\
12.2 / 100 \text {-person/months (95\% CI } 8.1- \\
17.8) \text { in the prophylactic dose group } \\
\text { and 20.1/100 person/month (95\% CI } \\
11-33.6 \text { ) } \\
\text { Anticoagulant sub-therapeutic dosages } \\
\text { are associated with an increased risk } \\
\text { of all-cause death (HR 3.89;95\% } 1.90 \text { - } \\
7.90 \text { ) }\end{array}$ \\
\hline
\end{tabular}


Table 2. continued

\begin{tabular}{|c|c|c|c|c|c|c|}
\hline Author/ Study & Intervention & Comparison & $\begin{array}{l}\text { Follow up } \\
\text { treatment follow } \\
\text { up }\end{array}$ & $\begin{array}{c}\text { Outcome } \\
\text { assessment }\end{array}$ & $\begin{array}{l}\text { Mortality } \\
\text { A vs. B (\%) }\end{array}$ & OR/HR \\
\hline $\begin{array}{l}\text { (Albani et al., } \\
\text { 2020) }\end{array}$ & $\begin{array}{l}\text { Enoxaparin: } 40-80 \mathrm{mg} \\
\text { in daily }\end{array}$ & without enoxaparin & $\begin{array}{l}\text { Patients were } \\
\text { tracked until } \\
\text { they died or were } \\
\text { discharged from } \\
\text { the hospital. }\end{array}$ & $\begin{array}{l}\text { the primary } \\
\text { o u t c o m e } \\
\text { was hospital } \\
\text { mortality }\end{array}$ & $\begin{array}{l}\text { No Enoxaparin: } 154(25.5 \\
\%) \\
\text { Enoxaparin: } 200(25 \%)\end{array}$ & $\begin{array}{l}\text { Compared to no enoxaparin treatment, } \\
\text { enoxaparin was related to decreased in- } \\
\text { hospital all-cause mortality (OR } 0.53 \text {, } \\
95 \% \text { CI } 0.40-0.70 \text {, with an E-value of } \\
2.08 \text { (upper CI 1.66). }\end{array}$ \\
\hline
\end{tabular}




\section{Table 2. continued}

\begin{tabular}{|c|c|c|c|c|c|c|}
\hline Author/ Study & Intervention & Comparison & $\begin{array}{l}\text { Follow up } \\
\text { treatment follow } \\
\text { up }\end{array}$ & $\begin{array}{l}\text { Outcome } \\
\text { assessment }\end{array}$ & $\begin{array}{l}\text { Mortality } \\
\text { A vs. B (\%) }\end{array}$ & OR/HR \\
\hline $\begin{array}{l}\text { (Shen et al., } \\
\text { 2021) }\end{array}$ & $\begin{array}{l}\text { Prophylactic LMWH: } \\
40 \mathrm{mg} \text { subcutan once } \\
\text { a day or } 40 \mathrm{mg} \text { every } \\
12 \text { hours }\end{array}$ & Without LMWH & $\begin{array}{l}\text { Patients were } \\
\text { tracked until } \\
\text { they died or were } \\
\text { discharged from } \\
\text { the hospital. }\end{array}$ & $\begin{array}{l}\text { Primary outcome: } \\
\text { in-hospital all- } \\
\text { cause mortality }\end{array}$ & $\begin{array}{l}\text { In-hospital mortality in } \\
\text { mild/moderate patients } \\
\text { was 0\% (LMWH) vs } 1.7 \% \\
\text { (Non LMWH), in severe } \\
\text { patient } 2.4 \% \text { (LMWH) vs } \\
18.8 \% \text { (non LMWH), and in } \\
\text { critically ill patient } 37.31 \% \\
\text { (LMWH) vs } 63.63 \% \text { (non } \\
\text { LMWH) }\end{array}$ & $\begin{array}{l}\text { LMWH was linked to decreased all- } \\
\text { cause death (Adjusted OR } 0.2 ; 95 \% \\
\text { CI, 0.09-0.46) than non-LMWH. } \\
\text { In subgroup analysis, LMWH was } \\
\text { related with lower death in severe } \\
\text { (adjusted OR 0.08, 95\% CI 0.01- } \\
0.06 \text { ) and critical patients (Adjusted } \\
\text { OR } 0.32 ; 95 \% \text { CI } 0.10-0.996 \text { ) }\end{array}$ \\
\hline
\end{tabular}

et The standard dose High dose

of Patients

were Primary outcome: OR $0.32 ; 95 \%$ CI $0.10-0.996$ )

of prophylactic prophyla ctic follow e d in-hospital

enoxaparin: $40 \mathrm{mg}$ enoxaparin :

once a day, increased $0.7-1 \mathrm{mg} / \mathrm{kg}$ twice (c umulative

to $60 \mathrm{mg}$ a day in daily

days) or

discharge from

hospital or change

in antithrombotic

therapy

(Zheng et al., Thromboprophylactic N

2021) e n o x a p a r i n : thromboprophylaxis enoxaparin was given

- Estimation of ICU Primary outcome: Non-survivor

incidence of Patients receiving standarddeath/in-hospital mortality dose enoxaparin had a decreased in standard dose $23.6 \%$ ) vs. cumulative incidence of mortality at $40.2 \%$ in the higher dose. 21 days than the higher dose (adjusted once a day at a dose of $100 \mathrm{IU} \mathrm{Axa} / \mathrm{kg}$ for at survival rate: 30 - survival day survival after mortality in ICU $21.67 \%$ vs $27.78 \%$ (non- no-thromboprophylaxis group (OR admission to the patient thromboprophylaxis) 0.60; 95\% CI 0.32-1.01)

least 5 days

For COVID-19

inpatients with VTE

suspects:100 IU Axa/

$\mathrm{kg}$ twice a day 
Table 2. continued

\begin{tabular}{|c|c|c|c|c|c|c|}
\hline Author/ Study & Intervention & Comparison & $\begin{array}{l}\text { Follow up } \\
\text { treatment follow } \\
\text { up }\end{array}$ & $\begin{array}{l}\text { Outcome } \\
\text { assessment }\end{array}$ & $\begin{array}{l}\text { Mortality } \\
\text { A vs. B (\%) }\end{array}$ & OR/HR \\
\hline $\begin{array}{l}\text { (Farrar et al., } \\
\text { 2021) }\end{array}$ & $\begin{array}{l}\text { Per-protocol (received } \\
\text { thromboprophylaxis): } \\
\text { Standard prophylactic } \\
\text { doses could be } \\
\text { r e c o m m e } \mathrm{d} \mathrm{e} \mathrm{d} \\
\text { initially, the dose } \\
\text { could be escalated } \\
\text { based on patient- } \\
\text { specific characteristics } \\
\text { (admitted to the } \\
\text { Hospital consensus). } \\
\text { Specific dose not } \\
\text { mentioned in studies }\end{array}$ & $\begin{array}{l}\text { Off-protocol (other } \\
\text { than prophylactic } \\
\text { regiment) }\end{array}$ & $\begin{array}{l}\text { Patients were } \\
\text { tracked until } \\
\text { they died or were } \\
\text { discharged from } \\
\text { the hospital. }\end{array}$ & $\begin{array}{l}\text { Second ary } \\
\text { o u t c o m e: } \\
\text { a } 11-\mathrm{c} \text { a u e } \\
\text { mortality, } \\
\text { majorbleeding }\end{array}$ & $\begin{array}{l}\text { All-cause mortality in per- } \\
\text { protocol was significantly } \\
\text { lower }(6.3 \%) \text { than off- } \\
\text { protocol }(11.8 \%)\end{array}$ & $\begin{array}{l}\text { Mortality in off-protocol had a higher } \\
\text { death risk than on-protocol (OR: } 2.5 \text {; } \\
95 \% \text { CI } 1.36-4.56)\end{array}$ \\
\hline $\begin{array}{l}\text { (Rentsch et al., } \\
\text { 2021) }\end{array}$ & $\begin{array}{l}\text { Prophylactic } \\
\text { anticoagulant }\end{array}$ & No anticoagulant & $\begin{array}{l}\text { Patients were } \\
\text { tracked from the } \\
\text { time they were } \\
\text { admitted to the } \\
\text { hospital until the } \\
\text { earliest data on } \\
\text { outcomes were } \\
\text { available. }\end{array}$ & $\begin{array}{l}\text { The primary } \\
\text { outcome was } \\
\text { mortality at } \\
\text { 30-days }\end{array}$ & $\begin{array}{l}\text { The cumulative incidence } \\
\text { of mortality at } 30 \text { days } \\
\text { for individuals receiving } \\
\text { prophylactic anticoagulant: } \\
\text { agents those getting no } \\
\text { anticoagulant was } 14.3 \\
\text { percent vs. } 18.7 \text { percent }\end{array}$ & $\begin{array}{l}\text { Prophylactic anticoagulant was } \\
\text { associated with a lower risk of inpatient } \\
\text { mortality compared to no anticoagulant } \\
\text { use (aHR } 0.69 ; 95 \% \text { CI } 0,61-0,77 \text { ) }\end{array}$ \\
\hline
\end{tabular}




\section{Table 2. continued}

\begin{tabular}{|c|c|c|c|c|c|c|}
\hline Author/ Study & Intervention & Comparison & $\begin{array}{l}\text { Follow up } \\
\text { treatment follow } \\
\text { up }\end{array}$ & $\begin{array}{l}\text { Outcome } \\
\text { assessment }\end{array}$ & $\begin{array}{l}\text { Mortality } \\
\text { A vs. B (\%) }\end{array}$ & OR/HR \\
\hline $\begin{array}{l}\text { (Helms et al., } \\
\text { 2021) }\end{array}$ & $\begin{array}{l}\text { A prophylactic dose } \\
\text { of enoxaparin: } 60 \mathrm{mg} \\
\text { twice daily in obese } \\
\text { patients or UFH } 200 \\
\text { IU/ } \mathrm{kg} \text { once a day } \\
\text { in individuals with } \\
\text { creatinine } \\
\text { clearance (ClCr) less } \\
\text { than } 30 \mathrm{~mL} / \mathrm{min} \text {. }\end{array}$ & $\begin{array}{l}\text { Therapeutic dose: } 100 \\
\text { IU/kg twice day of } \\
\text { LMWH based on body } \\
\text { weight, not to exceed } \\
10,000 \mathrm{IU} / 12 \mathrm{~h} \text { or } 500 \\
\mathrm{IU} / \mathrm{kg} \text { daily of UFH } \\
\text { for individuals with } \\
\text { creatinine clearance } \\
(\mathrm{ClCr}) \text { less than } 30 \\
\mathrm{~mL} / \mathrm{min}\end{array}$ & $\begin{array}{l}\text { Follow-up for } \\
\text { the most recently } \\
\text { admitted patients } \\
\text { lasted } 80 \text { days. }\end{array}$ & $\begin{array}{l}\text { Mortality } \\
\text { ICU }\end{array}$ & $\begin{array}{l}\text { Prophylactic: } 20(18.5 \%) \\
\text { Therapeutic: } 11(15.5 \%)\end{array}$ & $\begin{array}{l}\text { Therapeutic dose treatment was } \\
\text { associated with decreased ICU mortality } \\
\text { than prophylactic dose treatment (OR } \\
0.84 ; 95 \% \text { CI } 0.43-1.13)\end{array}$ \\
\hline
\end{tabular}

Abbreviations: LMWH, Low Molecular Weight Heparin; UFH, Unfractionated Heparin; ICU, Intensive Care Unit; BMI, Body Mass Index; IU, International Unit; VTE, Venous Thromboembolism Event; IPTW, Inverse Probability of Treatment Weighted OD, Once Daily; BID, Bis In Die (Twice in Daily); TID, Ter In Die (Three times in daily); RBC, Red Blood Cell OR, Odds Ratio; HR, Hazard Ratio; CI, Confidence Interval; NR: Not Reported 
Shen et al. (2021) conclude that the use of prophylactic LMWH is related to decreased all-cause mortality when compared to no LMWH use (adjusted OR 0.2, 95\% CI 0.09-0.46; 525 participants) after adjusting for some covariates age, sex, severity classification and comorbidities (Shen et al., 2021). These results align with Albani et al. (2020) study of 1376 hospitalized patients, which reported that mortality in enoxaparin intervention was $25 \%$ and in the comparator group was $25.5 \%$. Enoxaparin is related to a decreased in-hospital mortality rate correlated to no enoxaparin usage (OR 0.53, 95\% CI 0.40-0.70) (Albani et al., 2020). Furthermore, the study conducted by Rentsch et al. (2021), with a larger sample size (4.297 patients), reported an association between the use of prophylactic anticoagulants and reduced death rates within 30 days when compared to no use of prophylactic anticoagulants after multivariate adjustment (aHR 0,69; 95\% CI 0,61-0,77) (Rentsch et al., 2021). In addition, Zheng et al. (2021) compared the thromboprophylaxis effect of enoxaparin versus no thromboprophylaxis using an ICU patient setting and assessing 30-day survival after ICU admission. The group of patients who received thromboprophylaxis had a lower 30-day mortality rate than the group who did not get thromboprophylaxis $(21.67 \%$ vs. $27.78 \%)$, thromboprophylaxis group had a lower mortality rate than the non-thromboprophylaxis group (unadjusted OR $0.60,95 \%$ CI $0.32-1.01 ; 180$ participants) (Zheng et al., 2021).

\section{Anticoagulant prophylaxis dose versus anticoagulant therapeutic dose}

Eight studies compared the use of different doses of anticoagulants. (Canoglu \& Saylan, 2020; Farrar et al., 2021; Grandone et al., 2021; Helms et al., 2021; Jonmarker et al., 2020; Martinelli et al., 2021; Mennuni et al., 2021; Pesavento et al., 2020). The definition of the anticoagulant dose, either standard prophylactic, intermediate or therapeutic, was varied in each study (Table 2). Most studies used prophylactic anticoagulant systemic therapy LMWH/enoxaparin. Some of the standard prophylactic doses reported included enoxaparin $40 \mathrm{mg}$ once daily or $0.5 \mathrm{mg}$ per $\mathrm{kg}$ twice a day, UFH $5000 \mathrm{IU}, 2.5 \mathrm{mg}$ of fondaparinux, 2500-5000 IU dalteparin, and 2500-4500 Itinzaparin (Table 2) (Albani et al., 2020; Canoglu \& Saylan, 2020; Jonmarker et al., 2020; Pesavento et al., 2020; Shen et al., 2021).

Pesavento et al. (2020) reported the study in 324 hospitalized patients that "all-cause mortality" at standard dose prophylaxis (12.2 per 100 person/month; 95\%CI 8.1-17.8) was lower than subtherapeutic doses (20.1 per 100 persons/month; 95\% CI 11.0-33.8) (Pesavento et al., 2020). These results are supported by research from Grandone et al. (2021) in 264 hospitalized patients; they reported that prophylactic LMWH administration significantly reduced mortality when compared to higher doses (OR 0.31 , 95\% CI 0.130.85) (Grandone et al., 2021) Furthermore, Mennuni et al. (2021) reported that prophylactic doses of LMWH were associated with lower in-mortality risk when compared to greater dosages (Adjusted OR 0.847; 95\% CI 0.400-1.792; 436 participants) (Mennuni et al., 2021). Martineli et al. (2021) also reported results that the standard prophylactic enoxaparin had a lower percentage of mortality incidence than the higher dose group (adjusted HR 0.39; 95\%CI 0.18-0.76) (Martinelli et al., 2021) However, these results are in contrast to those of Canoglu \& Saylan. (2020), who reported that prophylactic doses of LMWH had a higher risk of death than therapeutic doses (OR 6,495; 95 percent CI 2,39317,627; 154 participants) (Canoglu \& Saylan, 2020).

Jonmarker et al. (2020) and Helms et al. (2021) discovered that anticoagulant use was related to a survival advantage in ICU patients (Helms et al., 2021; Jonmarker et al., 2020). In Jonmarker et al. (2020) study reported that lower risk of ICU mortality was associated with higher dose thromboprophylaxis, both in highdose thromboprophylaxis (HR 0.33 ; 95\%CI $0.13-0.87$ ) and medium-dose thromboprophylaxis (HR 0.88; 95\% CI 0.43-1.83) compared to patients who received lowdose thromboprophylaxis (Jonmarker et al., 2020). These results are in line with Helms et al. (2021); their study of 179 ICU patients also reported that prophylactic anticoagulant administered to them resulted in higher mortality compared to therapeutic doses (18.5\% vs.15.5\%) (Helms et al., 2021).

In another study, Farrar et al. (2021) compared an onprotocol group (receiving prophylactic anticoagulant according to the protocol) and an off-protocol group, reporting a higher incidence of death in the latter than the former (OR: 2.5 ; 95 percent CI 1.36-4.56) in patients with D-dimer 1500ng/ml (Farrar et al., 2021).

\section{Qualitative Review: The Anticoagulant Safety Profile in COVID-19 Patients}

Safety assessment of the use of anticoagulants must also be considered when treating COVID-19 patients. Major bleeding events are one of the most frequent adverse effects of using systemic anticoagulants. Studies have reported various types of bleeding events (both major and non-major) in COVID-19 hospitalized patients receiving anticoagulant Treatment (Albani et al., 2020; Farrar et al., 2021; Grandone et al., 2021; Helms et al., 2021; Jonmarker et al., 2020; Martinelli et al., 2021; Mennuni et al., 2021; Pesavento et al., 2020; Rentsch et al., 2021; Shen et al., 2021), apart from two studies that did not assess such events (Ćanoglu \& Saylan, 2020; Zheng et al., 2021). 
A. Anticoagulation prophylaxis versus no anticoagulant

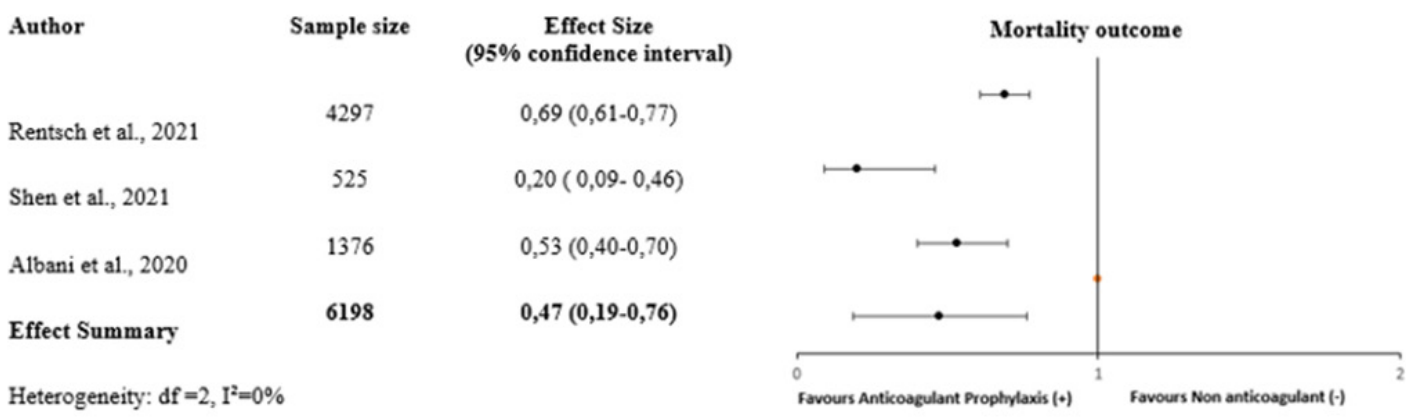

B. Prophylaxis dose anticoagulant versus Intermediate-to-therapeutic dose anticoagulation

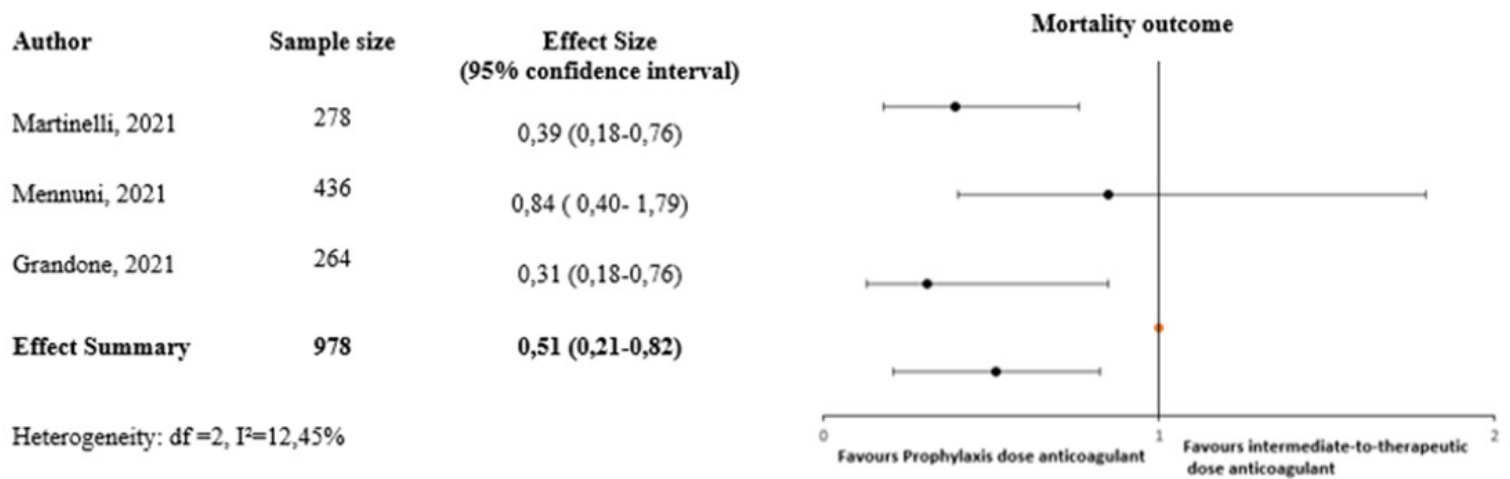

Figure 2. The Pooled Effect Size of mortality among COVID-19 patients in hospitalization with anticoagulant: (A) prophylaxis anticoagulant compared to patients without anticoagulant use, (B) Prophylaxis dose compared to intermediate-to-therapeutic dose anticoagulant, abbreviation: df, degree of freedom

Most studies report a higher incidence of bleeding with higher prophylactic doses of anticoagulants (intermediate/sub-therapeutic) than standard-dose prophylaxis (Grandone et al., 2021; Jonmarker et al., 2020; Martinelli et al., 2021; Mennuni et al., 2021; Pesavento et al., 2020). Pesavento et al. (2020) reported that the incidence of significant bleeding in the (sub) therapeutic dose group was higher $(9.5 \%)$ than the standard prophylactic dose group (3.3\%) (Pesavento et al., 2020). Jonmarker et al. (2020) also show similar results; the proportion of bleeding events in low dose prophylactic was lower (11.9\%) than the higher dose prophylactic $(14.6 \%)$, respectively $(\mathrm{p}=0.16)$ (Jonmarker et al., 2020). These results align with Mennuni et al. (2021); they reported a relatively low incidence of bleeding events in both groups. Major bleeding in the prophylaxis dose regimen was lower than the higher dose regimen, although the difference was insignificant (Mennuni et al., 2021). Furthermore, the research by Martinelli et al. (2021) also reported that major bleeding happened in $4 / 127$ patients $(3.1 \%)$ in higher dose enoxaparin, while no bleeding events were observed in the standard dose prophylactic group (Martinelli et al., 2021).
Several other studies reported bleeding events in the group receiving anticoagulants compared to those without anticoagulants (Albani et al., 2020; Farrar et al., 2021; Rentsch et al., 2021; Shen et al., 2021). Rentsch et al. (2021) report no risk of severe bleeding events; according to the results of a post hoc safety test, prophylaxis anticoagulant was not related to a higher incidence of bleeding with a need for transfusion when compared to non-anticoagulant use and bleeding events that required transfusion was rare $(4.6 \%)$ (Rentsch et al., 2021). The study of Shen et al. (2021) also reported no statistical difference in the major bleeding event between individuals receiving LMWH anticoagulants (2 patients) and those who did not (1 patient) (Shen et al., 2021). Furthermore, the research conducted by Farrar et al. (2021) reported that the on-protocol group (receiving prophylactic therapy according to the protocol) had a considerably lower bleeding percentage than the offprotocol group $(3.1 \%$ vs. $9.6 \%, \mathrm{p}<0.001)$ (Farrar et al., 2021). Moreover, Albani et al. (2020) reported results, with its prophylactic enoxaparin group having a lower percentage of bleeding events than without enoxaparin $(1.2 \%$ vs. $2.5 \%)$. However, the difference was not significant (Albani et al., 2020). 


\section{Quantitative: Meta-Analysis}

Of the 12 research cited above, 3 were conducted in the ICU setting, while the other 9 were in the all-hospitalized setting (Helms et al., 2021; Jonmarker et al., 2020). Four of the 9 studies compared the use of prophylactic anticoagulants with non-anticoagulant usage (Albani et al., 2020; Farrar et al., 2021; Rentsch et al., 2021; Shen et al., 2021). The other 5 studies compared the use of standard prophylactic dose anticoagulants to higher dose (intermediate-to-therapeutic) anticoagulants (Canoglu \& Saylan, 2020; Grandone et al., 2021; Martinelli et al., 2021; Mennuni et al., 2021; Pesavento et al., 2020).

Six observational studies with 7.176 hospitalized COVID-19 patients were involved in the quantitative meta-analysis with some considerations (Albani et al., 2020; Grandone et al., 2021; Martinelli et al., 2021; Mennuni et al., 2021; Rentsch et al., 2021; Shen et al., 2021). We performed to compare prophylactic anticoagulant to without anticoagulant used. Moreover, we independently considered the association of heparinoid anticoagulant at prophylactic doses with in-hospital mortality compared to intermediate-totherapeutic dose anticoagulant. Based on the pooling effect size results, the prophylactic dose of anticoagulant significantly reduces the risk of mortality by $40-50 \%$. Administration of prophylactic doses of anticoagulants decreased mortality compared with no anticoagulants and intermediate-to-therapeutic anticoagulants.

In comparison between prophylactic anticoagulant versus no anticoagulant use, we performed a combined meta-analysis of 3 studies. Farrar et al. (2021) should be excluded because of methodological differences, and the adjusted association of anticoagulants to the use of anticoagulants in-hospital mortality was not reported. By pooling the result of the three studies on all COVID-19 patients, the risk of in-hospital mortality was reduced by $53 \%$ (pooled OR $0.47 ; 95 \%$ CI $0.19-0.76 ; \mathrm{I}^{2}=0 \%$ ) (Figure 2A). In addition, compared with intermediateto-therapeutic dose anticoagulants, prophylactic dose anticoagulants were also associated with decreased risk of in-hospital mortality in COVID-19 patients hospitalized (pooled OR 0.51; 95\% CI 0.21-0.82, $\mathrm{I}^{2}=12.4 \%$, three studies, $\mathrm{N}=978$ ) (Figure 2B). Canoglu \& Saylan (2020) and Pesavento et al. (2020) were excluded from the meta-analysis because they reported different methodological and the adjusted effect size.

In our study, we could not perform a pooling metaanalysis of 3 studies in the ICU setting. This is due to the absence of reported adjusted associations between anticoagulant use and in-hospital all-cause mortality in the study of Helms et al. and Zheng et al., which prevented the pooling of data, so data were synthesized narratively. The pooling meta-analysis could not be reported in assessing bleeding events because most studies did not report adjusted association anticoagulant use in bleeding events. They only reported the proportion of bleeding events in each study that prevented the pooling of data, and the data were synthesized narratively (in the Qualitative review section).

\section{DISCUSSION}

The present study's main finding is that using heparinoid as a prophylactic systemic anticoagulant was related to a substantially reduced risk of in-hospital mortality in COVID-19 patients who were hospitalized. For hospitalized COVID-19 patients, heparin was suggested in some treatment guidelines. Not only employed as an anticoagulant, but heparin can also be used as an antiviral and anti-inflammatory treatment. It can bind to the spike protein in SARS-CoV-2 and act as a viral inhibitor to break down infectiousness (Buijsers et al.,2020). Previous in vitro studies reported that the SARS-CoV-2 spike protein interacts with a greater affinity for heparan sulfate than the SARS-CoV or MERS-CoV spike protein (Kim et al., 2020). Tree et al. (2021) also reported that UFH had a solid antiviral effect, with IC50 values ranging $25-41 \mu \mathrm{g} / \mathrm{ml}$, while LMWH had a lower inhibitory ability (IC50 values range $3.4-7.8 \mathrm{mg} / \mathrm{ml}$ ) (Tree et al., 2021). Heparin's ability to inhibit SARS-CoV-2 infection was reported to depend on the concentration used. Heparin was shown to effectively inhibit $70 \%$ of SARS-CoV-2 in Vero cells at a concentration of $100 \mathrm{mg} / \mathrm{ml}$ (MycroftWest et al., 2020). As an anti-inflammatory, heparin is reported to reduce NF-B signaling to the endothelium, resulting in decreased synthesis of pro-inflammatory cytokines such as TNF, IL6, and IL-1. This mechanism can be used to prevent the occurrence of cytokine storms, which can worsen the prognosis of COVID-19 patients (Hippensteel et al., 2020).

Heparin's potential as a commonly used anticoagulant has proven to be very strong (Albani et al., 2020; Farrar et al., 2021; Grandone et al., 2021; Mennuni et al., 2021; Rentsch et al., 2021; Shen et al., 2021). However, its optimal dose and the possibility of bleeding are of particular concern in its use. Research on the optimal dose of anticoagulants is still at the development stage. The risk of bleeding especially concerns the administration of anticoagulant therapy, and the occurrence of inpatient mortality is also associated with that of major bleeding after such therapy (Khan et al., 2020).

Most of the studies obtained results showing that prophylactic doses of anticoagulants reduced mortality (Grandone et al., 2021; Mennuni et al., 2021; Shen et al., 2021; Martinelli et al., 2021; Albani et al., 2020; Martinelli et al., 2021; Pesavento et al., 2020). Most of them are studied from Italy and Iran (study Pesavento et 
al.). However, two studies from the other country (study Canouglu et al. from Turkey and study Helms et al. from France) produced different results regarding the effect of prophylactic and therapeutic doses on mortality rates (Canoglu \& Saylan, 2020; Helms et al., 2021). Canoglu \& Saylan (2020) and Helms et al. (2021) reported that prophylactic doses of LMWH posed a higher risk than therapeutic doses of LMWH. Canoglu \& Saylan (2020) established $0.5 \mathrm{mg}$ per kg every 12 hours as a prophylactic dose and $1 \mathrm{mg}$ per kg every 12 hours as a therapeutic dose. On the other hand, Helms et al. (2021) used prophylactic doses of UFH 6000 IU twice a day subcutaneously in patients with obesity or UFH 200 IU per $\mathrm{kg}$ daily in creatinine clearance $(\mathrm{ClCr})$ less than 30 $\mathrm{mL} / \mathrm{min}$. Meanwhile, the therapeutic dose of LMWH is 100 IU per kg every 12 hours subcutaneously according to body weight, with a maximum dose of 10,000 IU twice every 12 hours or UFH 500 IU per kilogram daily for individuals with creatinine clearance less than $30 \mathrm{~mL} /$ min (Helms et al., 2021). However, most studies report that prophylactic doses have a lower risk of mortality than therapeutic ones.

The standard prophylactic doses reported include enoxaparin $40 \mathrm{mg}$ once daily (Grandone et al., 2021; Mennuni et al., 2021; Shen et al., 2021; Martinelli et al., 2021), enoxaparin $40 \mathrm{mg}-80$ once daily (Albani et al., 2020), enoxaparin $60 \mathrm{mg}$ twice daily in obese patients (Martinelli et al., 2021); and $2.5 \mathrm{mg}$ fondaparinux (Pesavento et al., 2020) (see Table 2). Apart from the definition of different doses, the differences in results may be influenced by other factors, including the essential characteristics of patients, their clinical condition, and standard of care in each country which are pretty diverse. While studies reviewed are ongoing, the standard of care for thromboprophylaxis use for COVID-19 patients was still very diverse. Although in April 2020, the International Society on Thrombosis and Haemostasis (ISTH) published recommendations for thromboprophylaxis with enoxaparin for COVID-19 patients, they specified the prophylactic dosage of LMWH as enoxaparin 4,000UI or $40 \mathrm{mg}$ subcutaneously every 12 hours. The evidence for this recommendation is inadequate, and evidence of a mortality advantage in individuals treated with enoxaparin is insufficient, primarily since COVID-19 is a novel disease (Thachil et al., 2020). The results of the studies reviewed can illustrate that standard prophylactic doses are shown to reduce patient mortality further. The standard dose commonly used in the studies reviewed is LMWH (enoxaparin) $40 \mathrm{mg}$ once daily.

The studies' results proved that treatment with a systemic prophylactic anticoagulant during hospital stay reduce themortality. Moreover, when prophylactic anticoagulants are taken on the general ward, the probability of ICU admission is reduced (Albani et al., 2020). Nevertheless, ICU patients need a high-dose anticoagulant because adjusted dosing is influenced by characteristic patients, including the risk of thromboembolic complications (Mennuni et al., 2021). There is a consensus reporting an increased risk of thromboembolic events in 20-43\% of COVID-19 patients admitted to ICUs despite receiving prophylactic anticoagulants. The most common occurrences are pulmonary embolism and vein thrombosis of the lower extremities (Pluta, Cieniewicz, \& Trzebicki, 2021). A high rate of thrombotic complications has been reported in patients with COVID-19 in the ICU. In a systematic screening report, many events of thrombotic complications would be missed by relying solely on clinical suspicion, so increasing the dose of thromboprophylactic anticoagulants in the ICU should be considered (Jenner et al., 2021).

In our meta-analysis, compared to the non-anticoagulant group, using a prophylactic dosage anticoagulant reduced the risk of in-hospital mortality by $53 \%$; this might imply that using a prophylactic dose anticoagulant as a part of COVID-19 treatments may be advantageous. In addition, standard-dose prophylactic heparinoid anticoagulant was associated with decreased in-hospital mortality more than intermediate-to-therapeutic dose anticoagulant (pooled OR 0.51; 95\% CI 0.21-0.82, $\mathrm{I} 2=12.4 \%$, three studies, $\mathrm{N}=978$ ). In contrast to the results of Kamel et al. (2021), they discovered that the prophylactic anticoagulant dosage might be related to increased in-hospital mortality compared to therapeutic anticoagulant dose (RR 1.58, $95 \%$ CI 1.34-1.87, three studies, N=963) (Kamel et al., 2021). These conflicting results are because of the wide variety of the study setting, populations, the patient clinical condition, type of healthcare, and therapeutic approaches. In addition, when studies reviewed are ongoing, the primary guidance has not yet established a standardized regimen for managing patients with COVID-19, so the standard of care for thromboprophylaxis use for COVID-19 patients in each study was still very diverse. To ascertain a more apparent optimal dose, it is necessary to standardize the definitions of standard, intermediate, and therapeutic doses and monitor patients' clinical conditions. The studies included had varying degrees of COVID-19 severity and suggested that heparinoid (especially LMWH) may benefit patients in the mild/moderate and severe ill COVID-19. These results are per the ISTH interim guidance, which recommends using LMWH in COVID-19 patients who have been hospitalized (Thachil et al., 2020). The American Society of Hematology (ASH) recently proposed using the prophylaxis anticoagulants doses rather than the intermediate and therapeutic anticoagulant doses in COVID-19 patients. However, this suggestion was based on evidence with a low degree of certainty (Cuker et al., 2021). 
In addition to the dose, the adverse effects of anticoagulants were also investigated, as bleeding needs to be considered in the administration of systemic anticoagulants. Bleeding remains a major safety concern when anticoagulant is administered in COVID-19 patients with coagulation disorders. Bleeding events, as measured by our study was relatively rare in anticoagulant therapy. Our result reported no statistically significant difference in major bleeding events between individuals receiving prophylactic anticoagulants and those who did not. Billet et al. (2020) reported that a higher probability of transfusion was not associated with any anticoagulant regimen (both prophylactic and therapeutic dose anticoagulant) (Billett et al., 2020). Jonmarker et al. (2020) stated no signs of higher bleeding in the critically ill patients with COVID-19 who received higher doses of anticoagulants; this could be due to the characteristics of patients who may not bleed easily even with larger doses (Jonmarker et al., 2020). According to our study, the incidence of the bleeding event was relatively low in both standard prophylactic dose group and intermediateto-therapeutic dosage, despite the proportion of bleeding event in standard prophylactic dose was lower than the higher dose. However, this risk does not lead to death, indicating that it can be safely used in this clinical setting. The studies reviewed to establish that there is potential for standard prophylactic anticoagulants to reduce mortality rates. However, their use needs to be made with intensive monitoring of therapy and side effects.

\section{LIMITATIONS}

This systematic review's strength is that it provides systematic information on the effects of heparinoids as systemic anticoagulants prophylactic on all-cause-death from COVID-19 in hospitals. It also evaluates the safety of therapy related to bleeding that has not previously been made. However, the study has limitations because the population engaged is limited (confined to a few regions and does not cover all countries and continents). All of the identified studies in our analysis were observational and retrospective; as a result, the association's strength could not be adequately determined. There are variances across studies that will lead to bias, such as differences in the setting (ICU and general wards), variations in the type of health facilities where the study took place; varying standards of care or protocol therapy in each country; and differences in dose and type of thromboprophylactic anticoagulant. Moreover, some studies did not report the exact prophylactic dose used. Furthermore, item scoring in quality assessment is still a subjective process that might lead to subjective bias. However, we have minimized subjective bias by independently assessing the study using the STROBE statement by two investigators, and the differences were resolved by discussion. The last, although the results of this meta-analysis were significant, extrapolation of these data studies should be done with caution because no randomized controlled trials were conducted on the systemic anticoagulants or LMWH included in this meta-analysis. Further research, including RCTs on the effects of systemic or heparinoid anticoagulants in patients with COVID-19, might help to explain and confirm the findings.

\section{CONCLUSION}

We discovered that using heparinoid as systematic prophylactic anticoagulant was associated with decreased in-hospital mortality in COVID-19 patients without an increase in bleeding events, despite the severity of their disease. The reduction in mortality associated with prophylactic heparinoid anticoagulant may be related to the prevention of the coagulopathy that commonly occurs in the development of COVID-19. Administration of prophylactic anticoagulants at higher doses (intermediate-to-therapeutic dose anticoagulant) benefits increasing survival rates, especially for ICU patients. However, the risk of bleeding is higher than with standard doses. Therefore, standard-dose prophylactic heparinoid anticoagulant (40 mg once daily of LMWH) should be considered for patients with non-critical or other conditions without contraindications. RCT clinical trials are required to establish conclusions about the potential effectiveness of this therapy in COVID-19 patients.

\section{DECLARATION}

\section{Author Contribution Categories:}

Conception/Design of study: Ariska Deffy Anggarany/ Familia Takhwifa

Data Analysis/Interpretation: Ariska Deffy Anggarany/ Rani Sauriasari/Muhammad Alkaff

Drafting Manuscript: Ariska Deffy Anggarany/Famila Takhwifa

Writing-review \& editing: Ariska Deffy Anggarany/ Famila Takhwifa/Rani Sauriasari/Hayatun Nufus/Diana Paramita

Critical Revision of Manuscript: Rani Sauriasari/ Muhammad Alkaff

Final Approval and Accountability: Rani Sauriasari

Technical or Material Support: Hayatun Nufus/Diana Paramita

Supervision: Rani Sauriasari

\section{ACKNOWLEDGMENT}

\section{Funding Statement}

The authors are grateful to the Directorate of Research and Development, Universitas Indonesia, for their financial support through PUTI Q1 Grant No. NKB1274/UN2.RST/HKP.05.00/2020. 


\section{CONFLICT OF INTEREST}

There are no conflicts of interest stated by the authors.

\section{REFERENCES}

Abou-Ismail, M. Y., Diamond, A., Kapoor, S., Arafah, Y., \& Nayak, L. (2020). The hypercoagulable state in COVID-19: Incidence, pathophysiology, and management. Thrombosis Research, 194(May), 101115. https://doi.org/10.1016/j.thromres.2020.06.029

Albani, F., Sepe, L., Fusina, F., Prezioso, C., Baronio, M., Caminiti, F., ... Natalini, G. (2020). Thromboprophylaxis with enoxaparin is associated with a lower death rate in patients hospitalized with SARS-CoV-2 infection. A cohort study. EClinicalMedicine, 27(April), 0-5. https:// doi.org/10.1016/j.eclinm.2020.100562

Bikdeli, B., Madhavan, M. V., Jimenez, D., Chuich, T., Dreyfus, I., Driggin, E., ... Lip, G. Y. H. (2020). COVID-19 and Thrombotic or Thromboembolic Disease: Implications for Prevention, Antithrombotic Therapy, and Follow-Up: JACC State-of-the-Art Review. Journal of the American College of Cardiology, 75(23), 2950-2973. https://doi.org/10.1016/j.jacc.2020.04.031

Billett, H., Reyes Gil, M., Szymanski, J., Ikemura, K., Stahl, L., Lo, Y., ... Bellin, E. (2020). Anticoagulation in COVID-19: Effect of Enoxaparin, Heparin and Apixaban on Mortality. SSRN Electronic Journal. https://doi. org/10.2139/ssrn.3679919

Buijsers, B., Yanginlar, C., Maciej-Hulme, M. L., de Mast, Q., \& van der Vlag, J. (2020). Beneficial nonanticoagulant mechanisms underlying heparin treatment of COVID-19 patients. EBioMedicine, 59. https://doi. org/10.1016/j.ebiom.2020.102969

Canoglu, K., \& Saylan, B. (2020). Therapeutic dosing of low-molecular-weight heparin may decrease mortality in patients with severe COVID-19 infection. Annals of Saudi Medicine, 40(6), 462-468. https://doi. org/10.5144/0256-4947.2020.462

Cuker, A., Tseng, E. K., Nieuwlaat, R., Angchaisuksiri, P., Blair, C., Dane, K., ... Schünemann, H. J. (2021). American Society of Hematology 2021 guidelines on the use of anticoagulation for thromboprophylaxis in patients with COVID-19. Blood Advances, 5(3), 872-888. https://doi.org/10.1182/bloodadvances.2020003763

Farrar, J. E., Trujillo, T. C., Mueller, S. W., Beltran, L., Nguyen, C., Hassell, K., \& Kiser, T. H. (2021). Evaluation of a patient-specific, targeted-intensity pharmacologic thromboprophylaxis protocol in hospitalized patients with COVID-19. Journal of Thrombosis and Thrombolysis, (0123456789). https:// doi.org/10.1007/s11239-021-02552-x

Flumingnan, R., Tinoco, J., Pascoal, P., Areias, L., Cossi, M., Fernandes, M., ... Matar, C. (2020). Prophylactic anticoagulants for people hospitalised with COVID-19. Cochrane Database of Systematic Reviews, (10). https://doi.org/10.1002/14651858.CD013739.www. cochranelibrary.com

Gómez-Mesa, J. E., Galindo-Coral, S., Montes, M. C., \& Muñoz Martin, A. J. (2021). Thrombosis and Coagulopathy in COVID-19. Current Problems in Cardiology, 46(3). https://doi.org/10.1016/j. cpcardiol.2020.100742

Grandone, E., Tiscia, G., Pesavento, R., De Laurenzo, A., Ceccato, D., Sartori, M. T., ... Vescovi, A. (2021). Use of low-molecular-weight heparin, transfusion and mortality in COVID-19 patients not requiring ventilation. Journal of Thrombosis and Thrombolysis, (0123456789). https:// doi.org/10.1007/s11239-021-02429-z

Helms, J., Severac, F., Merdji, H., Schenck, M., ClereJehl, R., Baldacini, M., ... Meziani, F. (2021). Higher anticoagulation targets and risk of thrombotic events in severe COVID-19 patients: bi-center cohort study. Annals of Intensive Care, 11(1). https://doi.org/10.1186/ s13613-021-00809-5

Hippensteel, J. A., LaRiviere, W. B., Colbert, J. F., Langou t-Astri, C. J., \& Schmidt, E. P. (2020). Heparin as a therapy for COVID-19: Current evidence and future possibilities. American Journal of Physiology - Lung Cellular and Molecular Physiology, 319(2), L211L217. https://doi.org/10.1152/AJPLUNG.00199.2020

Jenner, W. J., Kanji, R., Mirsadraee, S., Gue, Y. X., Price, S., Prasad, S., \& Gorog, D. A. (2021). Thrombotic complications in 2928 patients with COVID-19 treated in intensive care: a systematic review. Journal of Thrombosis and Thrombolysis, 51(3), 595-607. https:// doi.org/10.1007/s11239-021-02394-7

Jonmarker, S., Hollenberg, J., Dahlberg, M., Stackelberg, O., Litorell, J., Everhov, Å. H., ... Cronhjort, M. (2020). Dosing of thromboprophylaxis and mortality in critically ill COVID-19 patients. Critical Care, 24(1), 1-10. https://doi.org/10.1186/s13054-020-03375-7

Kamel, A. M., Sobhy, M., Magdy, N., Sabry, N., \& Farid, S. (2021). Anticoagulation outcomes in hospitalized Covid-19 patients: A systematic review and metaanalysis of case-control and cohort studies. Reviews in Medical Virology, 31(3). https://doi.org/10.1002/ rmv. 2180 
Khan, A., Althunayyan, S., Alsofayan, Y., Alotaibi, R., Mubarak, A., Arafat, M., ... Jokhdar, H. (2020). Risk factors associated with worse outcomes in COVID-19: A retrospective study in Saudi Arabia. Eastern Mediterranean Health Journal, 26(11), 1371-1380. https://doi.org/10.26719/emhj.20.130

Kim, S. Y., Jin, W., Sood, A., Montgomery, D. W., Grant, O. C., Fuster, M. M., ... Linhardt, R. J. (2020). Characterization of heparin and severe acute respiratory syndrome-related coronavirus 2 (SARSCoV-2) spike glycoprotein binding interactions. Antiviral Research, 181(May). https://doi.org/10.1016/j. antiviral.2020.104873

Klok, F. A., Kruip, M. J. H. A., Meer, N. J. M. van der, Arbous, M. S., Gommers, D. A. M. P. J., Kant, K. M., ... Endeman, H. (2020). Incidence of thrombotic complications in critically ill ICU patients with COVID-19. Thromb Res, (January). https://doi.org/ https://doi.org/10.1016/j.thromres.2020.04.013

Langer, F., Kluge, S., Klamroth, R., \& Oldenburg, J. (2020). Coagulopathy in COVID-19 and Its Implication for Safe and Efficacious Thromboprophylaxis. Hamostaseologie, 40(3), 264-269. https://doi. org/10.1055/a-1178-3551

Martinelli, I., Ciavarella, A., Abbattista, M., Aliberti, S., De Zan, V., Folli, C., ... Peyvandi, F. (2021). Increasing dosages of low-molecular-weight heparin in hospitalized patients with Covid-19. Internal and Emergency Medicine, 16(5), 1223-1229. https://doi.org/10.1007/ s11739-020-02585-9

Mennuni, M. G., Renda, G., Grisafi, L., Rognoni, A., Colombo, C., Lio, V., ... Patti, G. (2021). Clinical outcome with different doses of low-molecular-weight heparin in patients hospitalized for COVID-19. Journal of Thrombosis and Thrombolysis, (0123456789). https:// doi.org/10.1007/s11239-021-02401-x

Mycroft-West, C. J., Su, D., Pagani, I., Rudd, T. R., Elli, S., Gandhi, N. S., ... Skidmore, M. A. (2020). Heparin Inhibits Cellular Invasion by SARS-CoV-2: Structural Dependence of the Interaction of the Spike S1 Receptor-Binding Domain with Heparin. Thrombosis and Haemostasis, 120(12), 1700-1715. https://oi. org/10.1055/s-0040-1721319

Nadkarni, G. N., Lala, A., Bagiella, E., Chang, H. L., Moreno, P., Pujadas, E., ... Fuster, V. (2020). Anticoagulation, Mortality, Bleeding and Pathology Among Patients Hospitalized with COVID-19: A Single Health System Study. Journal of the American College of Cardiology. https://doi.org/10.1016/j.jacc.2020.08.041 Neyeloff, J. L., Fuchs, S. C., \& Moreira, L. B. (2012).
Meta-analyses and Forest plots using a Microsoft Excel spreadsheet: Step-by-step guide focusing on descriptive data analysis. BMC Research Notes, 5(Mix). https://doi. org/10.1186/1756-0500-5-52

Page, M. J., McKenzie, J. E., Bossuyt, P. M., Boutron, I., Hoffmann, T. C., Mulrow, C. D., ... Moher, D. (2021). The PRISMA 2020 statement: An updated guideline for reporting systematic reviews. The BMJ, 372. https://doi. org/10.1136/bmj.n71

Patel, N. G., Bhasin, A., Feinglass, J. M., Belknap, S. M., Angarone, M. P., Cohen, E. R., ... Bashyam, M. D. (2020). Clinical Outcomes of Hospitalized Patients with COVID-19 on Therapeutic Anticoagulants. medRxiv, 1-30. https://doi.org/10.1101/2020.08.22.20179911

Pesavento, R., Ceccato, D., Pasquetto, G., Monticelli, J., Leone, L., Frigo, A., ... Vettor, R. (2020). The hazard of (sub)therapeutic doses of anticoagulants in noncritically ill patients with Covid-19: The Padua province experience. Journal of Thrombosis and Haemostasis, 18(10), 2629-2635. https://doi.org/10.1111/jth.15022

Pluta, J., Cieniewicz, A., \& Trzebicki, J. (2021). COVID-19: Coagulation disorders and anticoagulant treatment in patients hospitalised in ICU. Anaesthesiology Intensive Therapy, 53(2), 153-161. https://doi. org/10.5114/ait.2021.105783

Rentsch, C. T., Beckman, J. A., Tomlinson, L., Gellad, W. F., Alcorn, C., Kidwai-Khan, F., ... Freiberg, M. S. (2021). Early initiation of prophylactic anticoagulation for prevention of coronavirus disease 2019 mortality in patients admitted to hospital in the United States: Cohort study. The BMJ, 372. https://doi.org/10.1136/bmj.n311

Shen, L., Qiu, L., Liu, D., Wang, L., Huang, H., Ge, H., ... Zhou, N. (2021). The Association of Low Molecular Weight Heparin Use and In-hospital Mortality Among Patients Hospitalized with COVID-19. Cardiovascular Drugs and Therapy. https://doi.org/10.1007/s10557020-07133-3

Tang, N., Bai, H., Chen, X., Gong, J., Li, D., \& Sun, Z. (2020). Anticoagulant treatment is associated with decreased mortality in severe coronavirus disease 2019 patients with coagulopathy. Journal of Thrombosis and Haemostasis, 18(5), 1094-1099. https://doi.org/10.1111/ jth.14817

Thachil, J., Tang, N., Gando, S., Falanga, A., Cattaneo, M., Levi, M., ... Iba, T. (2020). ISTH interim guidance on recognition and management of coagulopathy in COVID-19. Journal of Thrombosis and Haemostasis, 18(5), 1023-1026. https://doi.org/10.1111/jth.14810 
Tree, J. A., Turnbull, J. E., Buttigieg, K. R., Elmore, M. J., Coombes, N., Hogwood, J., ... Carroll, M. W. (2021). Unfractionated heparin inhibits live wild type SARS-CoV-2 cell infectivity at therapeutically relevant concentrations. British Journal of Pharmacology, 178(3), 626-635. https://doi.org/10.1111/bph.15304

von Elm, E., Altman, D. G., Egger, M., Pocock, S. J., Gøtzsche, P. C., \& Vandenbroucke, J. P. (2014). The strengthening the reporting of observational studies in epidemiology (STROBE) statement: Guidelines for reporting observational studies. International Journal of Surgery, 12(12), 1495-1499. https://doi.org/10.1016/j. ijsu.2014.07.013

Weeks, L. D., Pharmd, K. W. S., Connors, J. M., \& Connell, N. T. (2021). Management of therapeutic unfractionated heparin in 19 patients : A retrospective cohort study. (March), 1-11. https://doi.org/10.1002/ rth2.12521
Zheng, R., Zhou, J., Song, B., Zheng, X., Zhong, M., Jiang, L., ... Sang, L. (2021). COVID-19-associated coagulopathy: thromboembolism prophylaxis and poor prognosis in ICU. Experimental Hematology and Oncology, 10(1), 1-11. https://doi.org/10.1186/s40164021-00202-9

Zhou, F., Yu, T., Du, R., Fan, G., Liu, Y., Liu, Z., ... Cao, B. (2020). Clinical course and risk factors for mortality of adult inpatients with COVID-19 in Wuhan, China: a retrospective cohort study. The Lancet, 395(10229), 1054-1062. https://doi.org/10.1016/S01406736(20)30566-3

Zhu, N., Zhang, D., Wang, W., Li, X., Yang, B., Song, J., ... Tan, W. (2020). A Novel Coronavirus from Patients with Pneumonia in China, 2019. New England Journal of Medicine, 382(8), 727-733. https://doi.org/10.1056/ nejmoa2001017 